\title{
METHOD OF SPECTRAL ANALYSIS OF TRACTION CURRENT OF AC ELECTRIC LOCOMOTIVES
}

\author{
Sergey GOOLAK ${ }^{1}$, Viktor TKACHENKO ${ }^{2}$, Gintautas BUREIKA ${ }^{3 * \#}$, Gediminas VAIČIŪNAS ${ }^{4}$ \\ 1,2Dept of Traction Rolling Stock, State University of Infrastructure and Technologies, Kyiv, Ukraine \\ ${ }^{3,4}$ Dept of Mobile Machinery and Railway Transport, Vilnius Gediminas Technical University, Vilnius, Lithuania
}

Received 6 March 2020; revised 7 June 2020, 5 August 2020; accepted 16 September 2020

\begin{abstract}
An improved method for spectral analysis of traction current of an Alternating Current (AC) electric locomotive is considered in the article. A new method of spectral analysis considers the change in voltage in the catenary system as a non-deterministic, non-ergodic and non-Gaussian process. It has been established that higher voltage harmonics in the catenary system have a significant negative effect on the operation of non-traction railway consumers of electricity. In addition, electric locomotives operating in the same feeder zone have a mutual influence on each other. Electric railway transport is a source of higher voltage harmonics and strongly distorts the shape of the sinusoidal voltage of the catenary system, which are caused by the higher spectral components of the current in the electric locomotive traction drive circuit. These spectral components of the traction current arise in the traction drive circuit due to the nonlinearity of the current-voltage characteristics of the electronic devices of an electric locomotive, for example, a contact rectifier, a capacitor circuit of traction motors. Reactive power compensators are used in electric locomotives to eliminate components of higher harmonic traction current in the catenary system. Traditionally, spectral analysis in such systems is performed using Fourier methods. However, the determination of the spectral components of the traction current by the Fourier method for constructing a control system for a reactive power compensator is possible only if the process of voltage variation is a deterministic or ergodic Gaussian process. Otherwise, the application of Fourier transform methods will be incorrect. An analysis of the factors that affect voltage changes in the catenary system showed that this process is significantly different from the ergodic Gaussian process. Such factors include the following: the operating mode of the electric locomotives; number and total capacity of electric locomotives in one feeder zone; electric locomotives passing through feeder zones; instability of collection current. Thus, in the case under consideration, the application of the Fourier methods is incorrect for the analysis of the spectral components of the traction current. This affects the quality of compensation of the higher harmonic components of the traction current, and in some cases, the unstable operation of the control system of the active part of the reactive power compensator. Proposed scientific approach is based on the Levinson-Durbin linear prediction algorithm. On the one hand, this allows adapting the control system of the compensator to the voltage parameters of the catenary system. On the other hand, this allows taking into account the operating modes of electric rail vehicle with reactive power compensation. The construction of a compensator control system using the Levinson-Durbin algorithm significantly simplifies the synchronization scheme of the compensator and power circuits of the traction electric drive of AC electric locomotive. A comparison of the traditional method of spectral analysis, based on the Fast Fourier Transform (FFT), and the method, based on the Levinson-Durbin algorithm, proposed by the authors, showed the high efficiency of the latter.
\end{abstract}

Keywords: railway transport, AC electric locomotive, reactive power compensator, Fast Fourier Transform (FFT), Levinson-Durbin method.

\section{State-of-art works of determining the spectrum components of current in power circuits of the Alternating Current (AC) locomotives}

The hybrid compensator valves of reactive power were widely used in the electric locomotives power supply system's starting from the 8th decade of the last century (Parikh, Patel 2014; Bagwan et al. 2014). It is of vital im- portance to use the qualitative controlling algorithm for active part of the compensator valve. Quality of the controlling algorithm is determined by opportunity of compensation of the supply current higher harmonic components (Liudvinavičius et al. 2011). In light of this fact, it is important to emphasise one special circumstance. Without looking at big number of the existing algorithms of the reactive power compensator valves control, used in power

\footnotetext{
${ }^{*}$ Corresponding author. E-mail: gintautas.bureika@vilniustech.lt

\#Editor of the TRANSPORT - the manuscript was handled by one of the Associate Editors, who made all decisions related to the manuscript (including the choice of referees and the ultimate decision on the revision and publishing).
}

Copyright (C) 2020 The Author(s). Published by Vilnius Gediminas Technical University

This is an Open Access article distributed under the terms of the Creative Commons Attribution License (http://creativecommons.org/licenses/by/4.0/), which permits unrestricted use, distribution, and reproduction in any medium, provided the original author and source are credited. 
supply, the algorithms based on elimination of the higher harmonic components, are in high demand. In order to construct such algorithms, it is needed to determine the higher harmonic components of the currents with high accuracy, which are happening in circuits of the electric power user (Parikh, Patel 2014; Bagwan et al. 2014; Pishgahzadeh et al. 2015). In scientific works of Jain, Ahmad (2016) and Jadhav et al. (2016) it is confirmed that the area of research, devoted to determination of the higher harmonic components of the current and the voltage in the electric power user's chain, is currently dominant.

In the study by Kostin and Petrov (2011) it is shown that, the search for the optimal method of analysis of electric power processes in electric traction systems necessitates consideration of which electric power process is being investigated - a deterministic or a random process. If the form of voltages $u$ and currents $i$ is non-sinusoidal, it is advisable to use classical Fourier analysis for spectral analysis of processes in power systems. Classic Fourier transform methods could be correct when processes in power systems are stationary. Nonetheless, the actual time dependences of the current and the voltage on input and output of the railway substations, and also on the electrically propelled vehicles are the random-processes realization. The traction energy supply system during analysis of such electrical power processes is thought of as stochastic structure. It is considered unjustified to use the classical spectrum analysis to such processes. When it is necessary to analyse the electrical power processes in the stochastic structures, it is expected that using the probabilistic approaches is more reasonable.

The autocorrelation functions method, offered in the article by Kostin and Petrov (2011), may be used in computing the components of total power and evaluation of the other energy indicators in the electric traction systems only when the voltage and the currents are random ergodic processes. This method does not consider the cases when the voltages and the currents are being the random non-ergodic processes. Nonetheless, when Authors tried this method to determine higher harmonic components of the voltages and the currents in the pulling drive circuits, they met unbiased difficulties.

They particularly associated with random occurrence of some harmonic components in the spectrum, which are the outcomes of any transient process, for example, separation of the current collector, or frictional sliding of the wage wheels (Jericjan et al. 2016; Goolak et al. 2019; Gorobchenko et al. 2018).

Description of such restriction is described in the work of Nazarov, N. S. and Nazarov, O. N. (2015), devoted to the distinctive features of the rail electrically propelled vehicles traction currents spectrum analysis. The approach for electrically propelled vehicles traction currents harmonic components determination used in it with consideration of the transient processes in the electrically propelled vehicles pulling drive is based on using wavelet-analysis. When such algorithm was used, it allows us to bypass the restrictions, placed by the transient processes in electri- cally propelled vehicles pulling drive. For this reason, the solutions, received by Nazarov, N. S. and Nazarov, O. N. (2015) give us the opportunity to compose the algorithm, which controls the active part of the compensator valve of reactive power, based on generation of the higher "opposite harmonics" of the current flowing through drive of vehicle. Yet there is still an issue of sustainability, accuracy and speed of the algorithm. It is especially currently central in synchronization of the compensator valve with the pulling drive.

Development of method concerning determination of the voltages and the currents in the electric transport systems is in the work of Kostin and Shejkina (2015), in which the authors describes the methods of decomposing the currents and the voltages random functions into simple random expressions, containing no less than three random values. Despite the fact that finding of such problem is obviously correct and recommendations how to use the methods of decomposing the currents and the voltages random functions into simple random expressions, the accuracy of such method still remains debatable.

Comparative analysis of the spectrum analysis methods is in the work of Kakora and Grinkevich (2017). It confirms hat in the classical spectrum analysis there are two regular, nonetheless equivalent methods to determine the power spectrum density. The indirect method is built upon using the infinite sequence of the data values to compute the autocorrelation sequence, Fourier transform of which gives the searched power spectrum density. The direct method to determine power spectrum density is based on computing the square of the Fourier transform module for infinite sequence of data with using the appropriate statistical averaging. Resulting function, received without such averaging is called Sample spectrum. It is unsatisfactory due to statistical invalidity of the assessments, obtained by virtue of it, as root-mean-square error of such assessments can be compared in value with average value of assessment. Also in the same scientific work (Kakora, Grinkevich 2017) according to mathematical simulation results were compared classical methods of receiving the power spectrum density with adaptive method of minimum variance. Sometimes this method is called: maximum likelihood method. It was found that the method of minimum variance has much better spectral resolution comparing to the classical methods in analysis of short records and big signal-to-noise ratio. Improvement in spectral resolution allows using the signals with lesser spectral width. The shortfall of this method of minimum variance is the fact that it is inefficient in computation terms comparing to the classical methods and in small signal-tonoise ratio causes resolution loss. The method of minimal variance was further developed in the works (Zabarankin, Uryasev 2014; Širca 2016). The advantages of the assessment by method of minimum variance include the fact that it gives spectrum, in which the peaks heights are linearly proportional to power of sinusoidal wave, which are present in the analysed process. Minimum variance has a more descriptive informativeness near the origin of the 
estimation coordinates. It was received it by minimizing the variance of the process on the output of the narrowband filter, frequency response of which adapts to spectrum components of input process on every frequency, which is of interest to us. But the assessment performed by method of minimum variance is not the truth function of the power spectrum density, as unlike the truthful power spectrum density the area under the graphics of the minimum variance method assessment does not describe the total power of the process, which is being measured. The Fourier inverse transform, which corresponds to minimum variance method assessment, also does not coincide with autocorrelation sequence, which is used to obtain this assessment - this is how it differs from Autocorrelation Process (AP)-assessments, which have these properties.

The classical methods of the spectrum estimation are among the most stable methods of the spectrum estimation. They may be used in all the types of signals and noises, which possess stationary properties. The methods, alternating to them, namely the ones of high resolution power turn out to be stable only in case of non-timevarying signals restricted class. In the classical method they usually use the Fast Fourier Transform (FFT) algorithm, due to which they are the most efficient methods in computation among the available methods of the spectrum estimation. It should be also noted that assessments of the power spectrum density, obtained with their help, are linearly dependent on power of the sinusoid waves, present in the data, while in the alternative methods very often they do not provide the alternative link between the amplitudes of the spectrum and the power of these sinusoid waves (Rahi, Mehra 2014). The main disadvantage of the spectrum estimation classical methods is distorted effect of penetration on the side lobes due to impending measurement in them of the finite data sequences. Data processing by virtue of the window enables to weaken the effect of the side lobes, nonetheless, only thanks to resolution loss. The resolution power, which is provided by the classical methods, cannot exceed the value, reciprocal to the data length.

The power spectrum density is determined as FFT of infinite autocorrelation sequence. This relation between power spectrum density and autocorrelation sequence it is possible to consider as the random process second order statistic non-parametric description. It was approach the second order statistic non-parametric description by considering the time-series model, which corresponds to the random process, analysed. In this case the power spectrum density time-series model, first and foremost is a certain function of this model parameters, and not of the autocorrelation sequence. Researchers Sasikiran et al. (2014), Nychka et al. (2015) and Sykulski et al. (2016) describe one individual plan of the models, which are actuated by white noise process and have rational system functions. This class includes autoregressive process model, the moving-average process model and autoregressive moving-average process model. The output processes of this class' models have power spectrum density, which are completely described by virtue of parameters of white noise process model and variance.

When one of these three models was chosen (autoregressive process, moving-average process, autoregressive moving-average process), some previous information about the possible form of spectrum assessment was needed (Sasikiran et al. 2014; Nychka et al. 2015; Sykulski et al. 2016). The moving-average process model used in such cases is mentioned when the spectrums with deep zeros are needed (Zhuang et al. 2014; Petitjean et al. 2014; Bagnall, Lines 2014), nevertheless, without acute peaks described in research works of Petitjean et al. (2016); Loch et al. (2016).

Concerning the autoregressive moving-average process model - it can be used in both border-line cases. In the cases when each of three models is usable, to use the one with the least number of the parameters. It should be noted that the computation efforts for estimation of autoregressive process model are often significantly less than the ones necessary to assess the parameters of moving-average process and autoregressive moving-average process models. That is why autoregressive process model of time series is sometimes used even when it is not the model with the least number of the parameters.

From all the time-series models they mostly focus on autoregressive spectrum assessments. There are two reasons for that: (1) the autoregressive spectrums usually have the acute peaks; they often associate this with high spectrum resolution power; (2) the assessments of the autoregressive process parameters are receive as linear equations solutions (Sasikiran et al. 2014; Nychka et al. 2015; Sykulski et al. 2016). At the same time the assessments of the moving-average process and autoregressive movingaverage process parameters require non-linear equations solution (Petitjean et al. 2014, 2016; Bagnall, Lines 2014).

Scientists Sasikiran et al. (2014), Nychka et al. (2015) and Sykulski et al. (2016) defined that there are three ways to implement the autoregressive process method:

- assumption that the autocorrelation sequence is known;

- maximum entropy method;

- linear prediction method.

Since in reality the assumption that autocorrelation sequence is known, is not typical, they almost never use the first method.

The researches Wollstadt et al. (2014); Jurado et al. (2015) and Benedetto et al. (2015) showed that using the maximum entropy method is reasonable only the research process is Gauss one. In the other cases, it is reasonable to use the linear prediction method.

All the methods of autoregressive spectrum assessment are classified into two categories:

- the algorithms for data pack processing;

- the algorithms for serial data processing.

The researches performed in the works of Sasikiran et al. (2014); Nychka et al. (2015); Sykulski et al. (2016) showed that it is reasonable to use the block methods of assessment only when the volume of available data is ex- 
tremely limited, nonetheless, it is necessary to receive assessments with the best possible descriptions. When longer data records are available, we can use the whole range of the sequential estimation method to update the autoregressive parameters assessments, when each new readout from the data acquisition system in real-time mode when they occur. After that using these updated parameters we can construct new diagram of spectrum assessment is conceivable, if necessary. Such methods are especially useful to track the signals with parameters, which slowly change in time. The methods of sequential estimation in time are sometimes called adaptive algorithms, as they constantly adapt to the signal performance, even when it changes. The adaptive filtering algorithm for the higher harmonic components of the traction current is also shown in work of Gulak et al. (2014), however, in this article it is shown only conceptually. The term "adaptive" is used in spectrum analysis to differ the parametric methods of spectrum assessment from non-parametric ones. It is understood that the parametric methods "adapt" their parameters to the nature of the data. In non-parametric method, such adjustment of the parameters does not happen.

The sequential methods, which are used in autoregressive process parameters assessment, are classified into two categories:

- the simplest methods based on gradient approximation; among them there is well known minimum mean squares method;

- recursive least squares method; these algorithms provide the best performances compare with minimum mean squares method, nevertheless, only thanks to additional computation effort.

Researchers Raja, Chaudhary (2015) and Li et al. (2017) showed that adaptive minimum mean squares algorithm can be used to track the changes in time of the input signal statistics, if they change slowly enough comparing to speed of this algorithm's convergence. Averaging by time of the errors sequence, generated by minimum mean squares algorithm, provides receiving of white noise variance assessment, necessary to compute power spectrum density of autoregressive process.

In case of relatively short data records, the gradient adaptive minimum mean squares algorithm concedes $\mathrm{PH}$-algorithm by its performance and convergence speed. However, it is more functionally stable and less sensitive to incorrect numerical causality and effects, resulting from finite word length, than recursive least squares algorithm. Therefore, in those applications, where there is enough data and slower convergence is allowable, minimum mean squares method may turn out to be completely acceptable algorithm of sequential spectrum assessment.

Comparisons recursive least squares and minimum mean squares algorithms, performed in the works by Ding et al. $(2015,2017)$ and Li, Liu (2018), show that concerning the established (standard) value the recursive least squares algorithm is faster (never slower) than minimum mean squares algorithm. If there is a loud noise, in other words in the case when dispersion of Eigen values of the autocorrelation matrix is not big, recursive least squares and minimum mean squares algorithms have comparative convergence speeds. The inherent noise, which is generated during gradient assessment by minimum mean squares algorithm method, occurs in form of some movement of the filter input variance and some disarrangement of prediction filter parameters. To lower the level of movement and disarrangement considering the adaptation time constant is possible, nonetheless, this causes increase in time convergence of the minimum mean squares algorithm. In case of minimum mean squares algorithm, the convergence speed and noise because of disarrangement of the prediction filter parameters are interconnected so none of the performances can be improved, without worsening the other one.

If the recursive least squares and minimum mean squares algorithms are implemented by hardware to perform assessment in real-time mode, then the performances of these two algorithms may be effected by the quantization errors and rounding ones due to small length of machine word and using the arithmetic operations with floating point. The rounding errors may be cumulated and grow with upslope time till normal running of the algorithm is broken.

In case of the recursive least squares algorithm to limit the value of the cumulated error and save withstand ability of the filter is conceivable, although these results in worsening of prediction parameters computed values accuracy worsening. For normal running of the recursive least squares algorithm during imaging of the numbers to use no less than 10 binary digit bits is required. For normal running of the minimum mean squares algorithm, 7 binary digit bits are enough. Both algorithms have comparative characteristics, when using no less than 12 binary digit bits.

The principle of the recursive least squares algorithm is the Yule-Walter equation (Ding et al. 2015, 2017; Li, Liu 2018), solution of which gives the value of linear prediction filter coefficients. Among the diversity of solution methods for Yule-Walter equation for comparison to focus on two algorithms it is necessary - fast recursive least squares method (Gulak et al. 2014, Raja, Chaudhary 2015, Li et al. 2017) and Levinson-Durbin algorithm (Selvaperumal et al. 2016; Xiao et al. 2018). It is reasonable to use the fast recursive least squares algorithm in the case when big rate of response of the algorithm implementation is needed. However, the fact that the big computation error is cumulated during big data quantity processing, restricts usage of this algorithm. The electrical power processes spectrum components value change process in the electrical transport systems is much slower during Levinson-Durbin algorithm implementation. In order to determine the said spectrum components, if it is necessary to process big data quantity with high accuracy using the Levinson-Durbin algorithm is reasonable.

Despite the obvious advantages of using the LevinsonDurbin algorithm to assess the power spectrum density in the electrical transport systems, the mathematical ap- 
paratus and algorithms of its implementation are presented in the works of Selvaperumal et al. (2016); Xiao et al. (2018) - only in conceptual context. The authors of the mentioned works are confining only to general presentation of the mathematical apparatus.

\section{Definition of the spectral components of the traction current}

Arrangement of the conducted researches results allows believe that the existing approaches to determine electrical power processes spectrum components in the electrical transport systems are based on assumption that traction energy system is stochastic one. In other words, it is predicted that in order to find electrical power processes spectrum components in such systems to use the mathematical apparatus of the random processes is essential. It is obvious that such approaches allow receive the acceptable solutions only if the random processes are the ergodic Gauss ones. The implication is that violation of such conditions does not give the opportunity to receive the optimum solutions to determine the spectrum components of the currents and voltages in the electrical transport systems. This part of the problem may be resolved by developing the procedure algorithm linear prediction assessment power spectrum density in the mentioned systems, based on Levinson-Durbin algorithm. Then the spectral components of the currents of the traction drive system of the electric rolling stock can be found from the obtained estimate of the spectral power density of the traction current.

The task of this study is the possibility of synthesizing a linear prediction algorithm to obtain the spectral components of traction currents of electric rail vehicles.

In order to achieve this goal, it is necessary to solve the following objectives:

- develop the linear prediction algorithm based on Levinson-Durbin algorithm;

- receive the spectral components of the traction current;

- obtain the spectral components of this current for the known curve of traction current of the AC electric locomotive by Fourier transform;

- compare the obtained results of the components of the traction current spectrum obtained by two methods.

Any non-deterministic signal and dynamic system are usually the non-stationary objects, and this significantly complicates their analysis. The main problem, which occurs during the non-deterministic signals of different nature and dynamic systems analysis, is determination of their time and frequency responses. Although we can always determine certain time interval $\Delta t=N \cdot T$ (where: $T$ - sampling period; $N$ - number of readouts), on which the object parameters change not that fast. Such slice is called the interval of quasi-stationary state, and the object parameters on this interval are considered to be constant ones. The signal segment $x(n \cdot T)$ on the interval $\Delta t$ is called the envelope (where: $n$ - iteration).
If some parametric object model (signal or system) accurate enough for interval of quasi-stationary state is built, then we can use it in different cases, for example, in some controlling systems is conceivably.

The simplest are linear systems that are easily described by difference equations or in terms of the $Z$-transform (transfer-function). Time delays have a “-” sign for easy transition to the filter transfer-function, written in terms of the $Z$-transform. The simplest model in this sense is a recursive system of order $M-1$, which is represented by difference equations (Ding et al. 2015):

$$
y(n)=\sum_{i=1}^{N-1} b_{i} \cdot x(n-1)-\sum_{k=1}^{M-1} a_{k} \cdot y(n-k),
$$

where: $x(n)$ - an actuating signal; $y(n)$ - signal, which is being observed; $a, b$ - the transfer-function coefficients.

The most commonly used is a pole model whose numerator (the left sum of the difference equation) contains only one coefficient $b_{0}$ and the difference equation has the form (Ding et al. 2015):

$$
y(n)=b_{0} \cdot x(n)-\sum_{k=1}^{M-1} a_{k} \cdot y(n-k) .
$$

Let us assume that some linear model with transferfunction $H(z)$ (Ding et al. 2015) is affected by some actuating signal $x(n)$, and on its output a signal $y(n)$ is generated (Figure 1). The actuating signal $x(n)$ and the output a signal $y(n)$ have the form in terms of the $Z$-transform $X(z)$ and $Y(z)$, respectively. The parameters, in other words, the transfer-function coefficients are unknown.

It is necessary to find such coefficients $\left\{a_{k}\right\}$, which allow performing the equality - Equation (2) - on the interval of quasi-stationary state in the event of actuating signal $x(n)$ to be known. The actuating signal can be either impulse noise or white one.

In reality to predict the signal value $y(n)$ by its previous and next counts is needed. In the first case they indicate that prediction is performed forward, in the second backward (Figure 2) (Ding et al. 2015, 2017). The linear prediction is a computational procedure, which allows us to predict the future readouts (with certain accuracy) on certain linear aggregate $L$ of the previous $y(n-l), l=1$, $2, \ldots, L$, weighed non-deterministic signal. The segment of the signal with $L$ readouts (Figure 2 ) is developed by means of the window with length $L$ (usually by hamming window). The useful purpose of the linear prediction is in:

- in time domain -the future signal by its previous $L$ readout is assessed;

- in frequency domain - the spectrum signal, which is being researched on its segment (envelope) with length of $L$ readouts is assessed.

The results of the linear prediction mission are as follows: the coefficients of the adaptive linear digital filter are received, amplitude-frequency response, which to a pretty good approximation degree coincides with spectrum signal form in the envelope. 
The linear prediction mission can be formulated as follows. It is necessary to find the coefficients $\left\{a_{k}\right\}$ of a certain linear system, at the output of which one can observe the signal $y(n), l=1,2, \ldots, L$, provided that the input signal is $x(n)$. Is known the system itself is described by transferfunction of polar type $H(z)=b_{0} \cdot\left(1+\sum_{k=1}^{K} a_{k} \cdot z^{-k}\right)^{-1}$, in which $K$ - is a prediction order (Ding et al. 2017).

It is assumed that the actuating signal is a digital unit impulse $x(n)=u_{0}(n)$.

The approach to solution of the set problem is as follows in Figure 3.

According to the Equation (2) the signal $y(n)$ is determined by the difference equation:

$$
\begin{aligned}
& y(n)=b_{0} \cdot x(n)-\sum_{k=1}^{M-1} a_{k} \cdot y(n-k), \\
& n=1,2, \ldots, L .
\end{aligned}
$$

Therefore, the finite duration impulse response filter in cascaded manner with the target system is switched on. The filter possesses the transfer-function $A(z)=1+\sum_{k=1}^{K} \bar{a}_{k} \cdot z^{-k}$ (Ding et al. 2017). The coefficients of which are $\bar{a}_{k}$ equal to the coefficients $\bar{a}_{k}$, namely $\bar{a}_{k}=a_{k}$. The transfer-function of the obtained system will look as $H_{\text {total }}(z)=b_{0}=$ const, where: $b_{0}$ is amplification constant (Ding et al. 2017).

The finite duration impulse response filter with transfer-function $A(z)$ is called the linear prediction filter, $K$ is prediction order, and its coefficients $\bar{a}_{k}$ are the linear prediction coefficients. In actuality the coefficients $\bar{a}_{k}$ will differ from the precise meaning of the coefficients $a_{k}$, and for this reason the predicted signal meaning $\bar{y}(n)$ will differ from the precise one $y(n)$ on prediction error value (at $n>0$ ) (Ding et al. 2017):

$$
\varepsilon(n)=y(n)-\bar{y}(n)=2 y(n)-\sum_{k=1}^{K} \bar{a}_{k} \cdot y(n-k),
$$

which we call the remainder.

Equation (4) obtains the form in terms of transform:

$$
\begin{aligned}
& E(z)=Y(z)-\sum_{k=1}^{K} a_{k} \cdot Y(z) \cdot z^{-k} . \\
& X(z) \underset{\text { actuating signal }}{\longrightarrow} H(z) \underset{\text { observed signal }}{\longrightarrow} Y(z)
\end{aligned}
$$

Figure 1. The system linear model

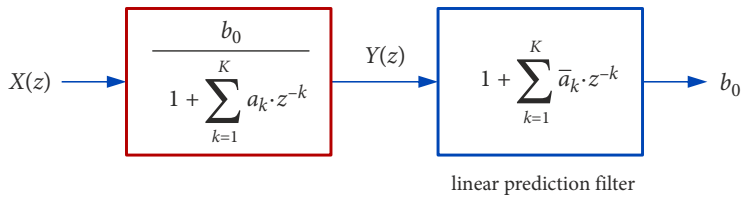

Figure 3. To the linear prediction problem solution (Selvaperumal et al. 2016; Xiao et al. 2018)

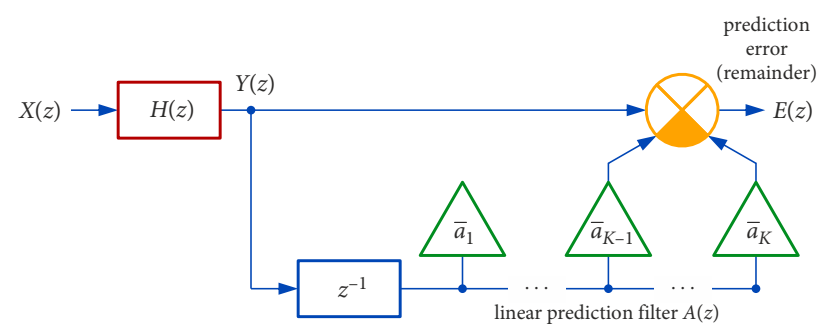

Figure 4 . The structure chart of the system with the linear prediction filter system

The remainder computation structure chart $E(z)$ is shown on Figure 4 (Li, Liu 2018). Therefore, the remainder is the output signal of the finite duration impulse response filter with transfer-function $A(z)$, in other words, on the linear prediction filter output.

The structure chart of the system and the Equation (5) show that, the transfer-function of the built system with the accuracy to the coefficient $b_{0}$ is a backwards transferfunction of the linear prediction filter $H(z)=b_{0} \cdot(A)^{-1}$.

In the following, it will be shown that linear prediction is a way of estimating the signal spectrum at the output of a linear system with unknown parameters. The focus at work is given to the deterministic case, when on the input of finite duration impulse response filter (Figure 4) of linear prediction order $K=M-1$ the known signal $Y(z)$ is acting, and on the output of the filter we have the remainder (prediction error) $E(z)$.

It is necessary that for all the $n=0,1,2, \ldots, L$, the remainder would be equal to zero $\varepsilon(n)=0$, in other words:

$$
y(n)-\underbrace{\bar{a}_{1} \cdot y(n-1)-\ldots-\bar{a}_{k} \cdot y(n-k)-\ldots-\bar{a}_{K} \cdot y(n-K)}_{\text {linear prediction filter }}=0 .
$$

Due to the fact that all the readouts of the signal $y(n)$ are known, with Equation (6) we get the linear equations system under zero-initial conditions for one envelope from $L$ readouts, provided that $L>>K$.

It should be noted that the following equation is obtained when the actuating signal $u_{0}(n)$ :

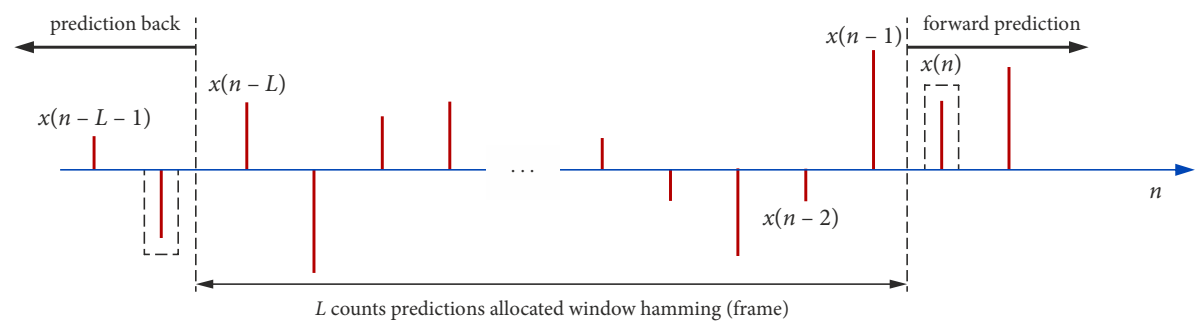

Figure 2. Prediction backward and forward (the appropriate readouts, which are predicted and outlined with dot lines) 


$$
\begin{aligned}
& \left.y(0)\right|_{x(n)=u_{0}(n)}=b_{0} ; \\
& \begin{cases}n=0 ; & \bar{a}_{1} \cdot(-1)+\ldots+\bar{a}_{k} \cdot(-k)+\ldots+\bar{a}_{K} \cdot(-K)=y(0)=b_{0} ; \\
n=1 ; & \bar{a}_{1} \cdot(0)+\ldots+\bar{a}_{k} \cdot(1-k)+\ldots+\bar{a}_{K} \cdot(1-K)=y(1) ; \\
\ldots & \ldots \\
n=l ; & \bar{a}_{1} \cdot(l-1)+\ldots+\bar{a}_{k} \cdot(l-k)+\ldots+\bar{a}_{K} \cdot(l-K)=y(l) ; \\
\ldots & \ldots \\
n=L ; & \bar{a}_{1} \cdot(L-1)+\ldots+\bar{a}_{k} \cdot(L-k)+\ldots+\bar{a}_{K} \cdot(L-K)=y(L) .\end{cases}
\end{aligned}
$$

The study of Xiao et al. (2018) shows the transition from a system of linear Equations (8) to an autocorrelation matrix, which has the form:

$$
\left[\begin{array}{ccccc}
R_{0} & R_{1} & R_{2} & \ldots & R_{K-1} \\
R_{1} & R_{0} & R_{1} & \ldots & R_{K-2} \\
R_{2} & R_{1} & R_{0} & \ldots & R_{K-3} \\
\ldots & \ldots & \ldots & \ldots & \ldots \\
R_{K-1} & R_{K-2} & R_{K-3} & \ldots & R_{0}
\end{array}\right] \cdot\left[\begin{array}{c}
a_{1} \\
a_{2} \\
a_{3} \\
\ldots \\
a_{K}
\end{array}\right]=\left[\begin{array}{c}
R_{1} \\
R_{2} \\
R_{3} \\
\ldots \\
R_{K}
\end{array}\right] .
$$

Autocorrelation coefficients are determined by expressions:

$$
\sum_{l=1}^{L} y(l-k) \cdot y(l-i)=R_{i k}
$$

where: $k$ is number of the column; $i$ is a number of the row. It is clear that all these amounts are the coefficients of autocorrelation with different relative shears (Ding et al. 2017). It is known that the coefficients of autocorrelation are the pair functions of time, in other words, their values depend on magnitude of difference in time $|i-k|$, so here we have the equality:

$R_{i k}=R_{k i}$.

Study (Xiao et al. 2018) also showed that:

- any diagonal parallel to the main one consists of the same coefficients $R_{j}$, since the difference in time $j=$ $k-i$ across the diagonal remains unchanged;

- the matrix remains symmetric because of the equality of the autocorrelation coefficients $R_{i k}=R_{k i}$.

The special structure of Equation (9) allows arranging the linear prediction parameters computation by means of the fast algorithms, among which the most popular one is Levinson-Durbin recurrent algorithm (Selvaperumal et al. 2016).

This algorithm is recurrent. The coefficients $a_{k}$ of the linear prediction filter order $K$ are computed in recurrent manner through the difference equations solution of the form (Equation (8)):

$$
R_{i}=\sum_{k=1}^{i} a_{k} \cdot R_{i-k}, i=1,2, \ldots, K,
$$

when the equation solution order $i$ is performed through the equation solution order $i-1$. This means that on the point $i$ is computing the prediction of the order $i$, in other words, the equations system - Equation (10) is computed logically for $i=1,2, \ldots, K$.
The prediction error square in the linear order prediction filter can be written as (Xiao et al. 2018):

$$
E_{i}=\sum_{l=1}^{L}\left(y_{i}(l)-\sum_{k=1}^{i} \bar{a}_{k}^{(i)} \cdot y_{i}(l-k)\right)^{2} \text {. }
$$

The other form of image for the means square error $E_{i}$ by inserting the determinations - Equation (10) into the Equation (13) is received:

$$
E_{i}=R_{0}-\sum_{k=1}^{i} \bar{a}_{k}^{(i)} \cdot R_{i-k}, i=1,2, \ldots, K
$$

with the implication that the total prediction error has two summands, from which one is constant, and the other depends on the prediction coefficients. It is also clear that first:

$$
E_{0}=R_{0}
$$

and second - from step to step, in other words, from the prediction of the order $i-1$ to the prediction of the order $i$, the total prediction error decreases:

$$
0 \leq E_{i} \leq E_{i-1} .
$$

In Levinson-Durbin algorithm on each step $i$ the two working parameters are computed:

- the total prediction error $E_{i}$ for the linear prediction filter of the order $i$ - Equation (14);

- the accessory parameter $r_{i}$, associated with the prediction error.

In the standard nominations Levinson-Durbin algorithm is as follows. The initial conditions are set - Equation (15).

In logical manner on the iteration step $i(l=1,2, \ldots$, $K)$ by using recurrent Equations (17)-(20) was computed:

$$
\begin{aligned}
& r_{i}=\frac{R_{i}-\sum_{k=1}^{i-1} a_{k}^{(i-1)} \cdot R_{i-k}}{E_{i-1}} ; \\
& a_{k}^{(i)}=r_{i} ; \\
& a_{k}^{(i)}=a_{k}^{(i-1)}-r_{i} \cdot a_{i-k}^{(i-1)}, 1 \leq k \leq i-1 ; \\
& E_{i}=\left(1-r_{i}^{2}\right) \cdot E_{i-1} .
\end{aligned}
$$

The prediction increases on one unit with each step, till achieving the value $K$. The terminal decision is determined on the step $K$ by relation:

$$
a_{K}=a_{K}^{(k)} \text { if } k=K \text {. }
$$

The accessory parameters $r_{i}$ in linear prediction theory are commonly referred to as reflection coefficients. The essence of the developed method for determining the components of the traction current spectrum in AC locomotives is as follows:

- on the input data series $x(n)$ the hamming window with length of $L$ is put;

- it is assumed that the linear system transfer-function $H(z)=b_{0} \cdot A(z)^{-1}$ equals to one; 
- the equations system - Equation (16) - for the input data $x(n)$ is composed;

- the prediction order $K$ was chosen and from the composed equations system the first block of size $(K \times K)$ for the data with the index from 0 to $K-1$ is isolated;

- for each equation of the first block data the prediction filter coefficients $a_{k}^{(i)}$ by means of LevinsonDurbin method and the value of the output signal by the expression was calculated:

$\bar{y}(n)=\sum_{k=1}^{K} a_{k}^{(i)} \cdot y(n-k) ;$

- proceeding to the next data block by increasing the index of each equations line in each equation on one;

- for each data block equation the prediction filter coefficients $a_{k}^{(i)}$ by virtue of Levinson-Durbin method was calculated; the output signal value by the Equation (22) only for the last equation of the block was defined;

- number of the processed blocks will equal to $L-K-1$;

- the spectrum components to receive the series of standard deterministic output signals by virtue of FFT were determined:

$\bar{Y}(k)=\sum_{n=0}^{L-1} \bar{y}(n) \cdot e^{-j \cdot n \cdot k \cdot \frac{2 \cdot \pi}{L}}$,

where: $k$ is the number of harmonic component; $\bar{Y}(k)$ is the value of the harmonic component $k$, imaged in complex form.

\section{Determination of the components of the traction current spectrum}

In the article by Kostin and Petrov (2011) the determining accuracy of the traction motors total power was compared using three methods: (1) FFT; (2) discrete electrical engineering; (3) correlation functions. All three cases considered the deterministic process of voltage change in the catenary system.

For the case of a non-deterministic process, a comparative measurement of the voltage in the catenary system was done using two methods: discrete electrical engineering and correlation functions. The calculation results were compared with the experimental results. It was concluded that the method of correlation functions has a smaller error than the method of discrete electrical engineering. It was recommended to use the method of correlation functions to study non-deterministic processes. The following conclusion was also made: if the spectra determined by two of the proposed methods coincide with the experiment for the case of a deterministic process, then the spectrum calculated by one of these methods will coincide for the case of a non-deterministic process as well.

The method of correlation functions underlies the Levinson-Durbin linear prediction algorithm. Therefore, the above conclusion is also true for this method. The Kostin and Petrov (2011) proposed calculating the spectral components of the traction current in two ways: according to the developed method and using the FFT for the case of determinism of the process of voltage change in the catenary system.

The article by Gulak et al. (2016) explores the traction current characteristic for an electric locomotive of the $\mathrm{VL}-80^{\mathrm{T}} \mathrm{K}$ series when driving along a straight section of the track at a constant speed of $46.8 \mathrm{~km} / \mathrm{h}$. The traction current curve is shown in Figure 5. In the time interval from 0 to $0.08 \mathrm{~s}$ there is a transient process of traction current, and in the time interval from 0.08 to $0.10 \mathrm{~s}$, the steady-state process. The spectral components of the traction current were determined for the steadystate process. The accuracy of the spectral analysis performed by the Fourier transform method depends on the number of samples of the function. The first 24 harmonic components were calculated at 128 input points. The sampling frequency was taken equal to the frequency of the first harmonic $-6400 \mathrm{~Hz}$. An order of 25 was selected for the prediction filter. The calculated complex values of the spectrum components were recorded in the form of the amplitude-frequency spectrum and phase-frequency spectrum. Comparative data of the spectral analysis of the traction current obtained by two methods - the authors' method and using the FFT - are presented in Table. As can be seen from Table, the difference in the results of spectral analysis performed by two different methods does not exceed $5 \%$.

This proves that the method developed by the authors for determining the spectral components of the traction current of an AC electric locomotive can be used for the algorithm for controlling the reactive power compensator.

Thus, the error in determining the spectral components of the traction current when using the author's method and, as a consequence, the error in generating the compensation current of the reactive power compensator, will not exceed the values allowed in engineering calculations.

However, the control circuit of the reactive power compensator, based on the algorithm for determining the spectral components by the method FFT, can adequately work only in the mode, when the voltage change in the traction power supply system is a deterministic process.

Moreover, the control circuit of the reactive power compensator, based on the spectrum determination algorithm proposed by the authors, can adequately work both in the mode when the change in the traction current voltage is a deterministic process, and in the mode when the voltages change is a non-deterministic process.

The obtained complex values of the spectrum components were recorded in the form of the amplitude-frequency and phase-frequency spectra.

The data comparing the results of calculating the components of the spectrum of the traction current curve, obtained using the method developed by the author and the FFT, are presented in Table. 


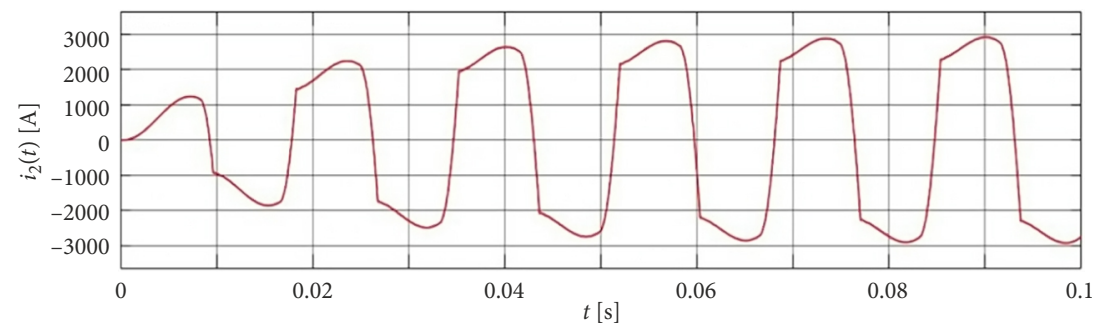

Figure 5. The traction current curve of $\mathrm{VL}-80^{\mathrm{T}}, \mathrm{K}$ series $\mathrm{AC}$ electric locomotive

Table. Comparison of computation results gained by developed method and the FFT

\begin{tabular}{|c|c|c|c|c|c|c|}
\hline \multirow{3}{*}{$\begin{array}{c}\text { No of } \\
\text { harmonic }\end{array}$} & \multicolumn{6}{|c|}{ Parameter } \\
\hline & \multicolumn{3}{|c|}{ Amplitude-frequency spectrum } & \multicolumn{3}{|c|}{ Phase-frequency spectrum } \\
\hline & The offered method [A] & FFT $[\mathrm{A}]$ & Difference [\%] & The offered method [rad] & FFT [rad] & Difference [\%] \\
\hline 0 & 39.259 & 39.342 & 0.211 & 3.142 & 3.141 & -0.032 \\
\hline 1 & 1442.309 & 1442.198 & -0.008 & -2.194 & -2.195 & 0.046 \\
\hline 2 & 31.565 & 31.459 & -0.305 & 1.982 & 1.981 & -0.050 \\
\hline 3 & 336.900 & 336.991 & 0.027 & -3.109 & -3.108 & -0.032 \\
\hline 4 & 27.079 & 27.155 & 0.033 & 1.036 & 1.038 & 0.193 \\
\hline 5 & 158.594 & 158.675 & 0.108 & 1.978 & 1.978 & 0.000 \\
\hline 6 & 17.744 & 17.657 & -0.493 & -0.103 & -0.107 & 3.738 \\
\hline 7 & 76.154 & 7.069 & -0.112 & 0.722 & 0.722 & 0.000 \\
\hline 8 & 10.040 & 9.975 & -0.652 & -1.584 & -1.570 & -0.892 \\
\hline 9 & 34.984 & 35.048 & -2.749 & -0.772 & -0.772 & 0.000 \\
\hline 10 & 7.555 & 7.658 & 1.345 & 2.927 & 2.935 & 0.273 \\
\hline 11 & 21.791 & 21.760 & -0.142 & -2.561 & -2.565 & 0.156 \\
\hline 12 & 7.428 & 7.423 & -0.067 & 1.435 & 1.423 & -0.843 \\
\hline 13 & 18.686 & 18.668 & -0.096 & 2.174 & 2.177 & 0.138 \\
\hline 14 & 6.260 & 6.171 & -1.442 & 0.042 & 0.043 & 2.326 \\
\hline 15 & 15.627 & 15.638 & 0.070 & 0.773 & 0.769 & -0.520 \\
\hline 16 & 4.908 & 4.994 & 1.722 & -1.819 & -1.792 & -1.507 \\
\hline 17 & 9.413 & 9.301 & -1.204 & -1.158 & -1.161 & 0.259 \\
\hline 18 & 4.908 & 4.69 & -4.648 & 2.543 & 2.537 & -0.236 \\
\hline 19 & 8.413 & 8.69 & 3.188 & -3.133 & -3.125 & -0.256 \\
\hline 20 & 5.524 & 5.441 & -1.525 & 0.977 & 0.962 & -1.559 \\
\hline 21 & 9.760 & 9.995 & 2.351 & 1.704 & 1.704 & 0.000 \\
\hline 22 & 5.577 & 5.862 & 4.862 & -0.471 & -0.463 & -1.728 \\
\hline 23 & 9.334 & 9.080 & -2.797 & 0.447 & 0.436 & -2.523 \\
\hline 24 & 5.683 & 5.724 & 0.716 & -1.814 & -1.826 & 3.305 \\
\hline
\end{tabular}

As can be seen from Table, the differences in the calculation of the components of the traction current spectrum, made using the method developed by the authors, do not exceed $5 \%$ of the data calculated using the FFT.

This proves that the developed method for determining the spectral components of the traction current of $\mathrm{AC}$ electric locomotives can be used in the development of the control system of the reactive power compensator. The difference in determining the spectral components of the traction current when using the method proposed by the authors, and, consequently, the difference in generating the compensating current caused by this circumstance, will not exceed the value allowed in engineering calculations. However, the control circuit of the reactive power compensator, based on the algorithm for determining the spectral components by the method - FFT, can adequately work only in the mode, when the voltage change in the traction power supply system is a deterministic process. While the control scheme of the reactive power compensator, based on the algorithm for determining the spectral proposed by the authors, can adequately work both in the mode when the voltage change in the traction power supply is a deterministic process and in the mode when the specified voltage change is non-deterministic. 


\section{Conclusions}

An improved method of spectral analysis for traction current of AC electric locomotive is proposed by the authors. The improvement is based on the principle of a linear prediction filter according to the Levinson-Durbin theory. The adequacy of proposed method is validated experimentally. This method allows modifying the control system of the reactive power compensator of $\mathrm{AC}$ electric locomotives and gaining the following results:

- optimized control of the compensator in the conditions of non-determinism of voltage changes in the catenary system;

- increased efficiency of reactive power compensation by adapting the compensator to the voltage of the traction energy system;

- increased power factor of the traction drive of the electric locomotive as a whole;

- increased stability of the compensator.

In addition, the proposed method significantly simplifies the synchronization system of the compensator and traction drive of AC electric locomotive.

\section{References}

Bagnall, A.; Lines, J. 2014. Technical report CMP-C14-01: an experimental evaluation of nearest neighbour time series classification, arXiv 1406.4757v1. Available from Internet: https://arxiv.org/abs/1406.4757

Bagwan, S. U.; Mulla, A. M.; Gudaru, U. 2014. Hardware circuit implementation of static VAR compensator (SVC) with thyristor binary compensator, International Electrical Engineering Journal 5(1): 1240-1246.

Benedetto, F.; Giunta, G.; Mastroeni, L. 2015. A maximum entropy method to assess the predictability of financial and commodity prices, Digital Signal Processing 46: 19-31. https://doi.org/10.1016/j.dsp.2015.08.001

Ding, F.; Wang, Y.; Ding, J. 2015. Recursive least squares parameter identification algorithms for systems with colored noise using the filtering technique and the auxiliary model, Digital Signal Processing 37: 100-108. https://doi.org/10.1016/j.dsp.2014.10.005

Ding, F.; Wang, Y.; Dai, J.; Li, Q.; Chen, Q. 2017. A recursive least squares parameter estimation algorithm for output nonlinear autoregressive systems using the input-output data filtering, Journal of the Franklin Institute 354(15): 6938-6955. http://doi.org/10.1016/j.jfranklin.2017.08.009

Gulak, S. A.; Slepuhin, A. Ju.; Chernyh, Ju. M.; Ermolenko, Je. K. 2014. Metod umen'shenija vysshih garmonik v naprjazhenii pitanija tjagovogo privoda jelektrovozov peremennogo toka s kollektornymi dvigateljami, Vestnik Belorusskogo gosudarstvennogo universiteta transporta: nauka i transport (1): 11-12. (in Russian).

Gulak, S. O.; Jermolenko, E. K. 2016. Model' systemy “tjagova pidstancija - kontaktna merezha - tjagovyj pryvid elektrovoza serii' VL-80 ${ }^{\mathrm{T}}$, K", Zbirnyk naukovyh prac' Derzhavnogo ekonomiko-tehnologichnogo universytetu transportu. Serija: Transportni systemy i tehnologii' 28: 99-109. (in Ukrainian).

Goolak, S.; Gerlici, J.; Tkachenko, V.; Sapronova, S.; Lack, T.; Kravchenko, K. 2019. Determination of parameters of asynchronous electric machines with asymmetrical windings of electric locomotives, Communications - Scientific Letters of the University of Zilina 21(2): 24-31.

https://doi.org/10.26552/com.C.2019.2.24-31

Gorobchenko, O.; Fomin, O.; Fomin, V.; Kovalenko, V. 2018. Study of the influence of electric transmission parameters on the efficiency of freight rolling stock of direct current, Eastern-European Journal of Enterprise Technologies 1(3): 60-67. https://doi.org/10.15587/1729-4061.2018.121713

Jadhav, S. S.; Mulla, A. M.; Gudaru, U. 2016. Performance realization of different reactive power controlling techniques: a review, International Journal of Advanced Research in Electronics and Communication Engineering 5(1): 29-34.

Jain, N.; Ahmad, A. 2016. Intelligent control scheme for mitigating voltage sag and swell problem in electrical power system using reactive power management, International Journal of Engineering Sciences \& Research Technology 5(8): 920-929. https://doi.org/10.5281/zenodo.60833

Jericjan, B. H.; Ljubars'kyj, B. G.; Jakunin, D. I. 2016. Modeljuvannja kombinovanoi' systemy nahylu kuzovu shvydkisnogo ruhomogo skladu zaliznychnogo transportu, Shidno-Jevropejskyj zhurnal peredovyh tehnologij 2(9): 4-17.

https://doi.org/10.15587/1729-4061.2016.66782 (in Ukrainian).

Jurado, S.; Nebot, À.; Mugica, F.; Avellana, N. 2015. Hybrid methodologies for electricity load forecasting: Entropy-based feature selection with machine learning and soft computing techniques, Energy 86: 276-291.

https://doi.org/10.1016/j.energy.2015.04.039

Kakora, V. A.; Grinkevich, A. V. 2017. Sravnitel'nyj analiz razreshajushhej sposobnosti algoritmov spektral'nogo ocenivanija, Doklady BGUIR 3: 20-24. (in Russian).

Kostin, N. A.; Petrov, A. B. 2011. Metody opredelenija sostavljajushhih polnoj moshhnosti v sistemah jelektricheskoj tjagi, Tehnicheskaja jelektrodinamika 3: 53-59. (in Russian).

Kostin, N. A.; Shejkina, O. G. 2015. Nekanonicheskoe spektral'noe razlozhenie sluchajnyh funkcij tjagovyh naprjazhenija i toka v sistemah jelektricheskogo transporta, Elektrotehnika i Elektromehanika (1): 68-71. (in Russian).

Li, M.; Liu, X. 2018. The least squares based iterative algorithms for parameter estimation of a bilinear system with autoregressive noise using the data filtering technique, Signal Processing 147: 23-34. https://doi.org/10.1016/j.sigpro.2018.01.012

Li, M.; Liu, X.; Ding, F. 2017. Least-squares-based iterative and gradient-based iterative estimation algorithms for bilinear systems, Nonlinear Dynamics 89(1): 197-211.

https://doi.org/10.1007/s11071-017-3445-x

Liudvinavičius, L.; Lingaitis, L. P.; Bureika, G. 2011. Investigation on wheel-sets slip and slide control problems of locomotives with ac traction motors, Eksploatacja i Niezawodność - Maintenance and Reliability 4: 21-28.

Loch, H.; Janczura, J.; Weron, A. 2016. Ergodicity testing using an analytical formula for a dynamical functional of alphastable autoregressive fractionally integrated moving average processes, Physical Review E 93(4): 043317. https://doi.org/10.1103/PhysRevE.93.043317

Nazarov, N. S.; Nazarov, O. N. 2015. Osobennosti spektral'nogo analiza tjagovyh tokov jelektropodvizhnogo sostava zheleznyh dorog, Sovremennye problemy sovershenstvovanija raboty zheleznodorozhnogo transporta 11: 88-100. (in Russian).

Nychka, D.; Bandyopadhyay, S.; Hammerling, D.; Lindgren, F.; Sain, S. 2015. A multiresolution Gaussian process model for the analysis of large spatial datasets, Journal of Computational and Graphical Statistics 24(2): 579-599.

https://doi.org/10.1080/10618600.2014.914946 
Parikh, R. S.; Patel, A. R. 2014. A user friendly Simulink model for FC-TCR to investigate power system issues, International Journal for Scientific Research \& Development 1(12): 26532656.

Petitjean, F.; Forestier, G.; Webb, G. I.; Nicholson, A. E.; Chen, Y.; Keogh, E. 2014. Dynamic time warping averaging of time series allows faster and more accurate classification, 2014 IEEE International Conference on Data Mining, 14-17 December 2014, Shenzhen, China, 470-479. https://doi.org/10.1109/ICDM.2014.27

Petitjean, F.; Forestier, G.; Webb, G. I.; Nicholson, A. E.; Chen, Y.; Keogh, E. 2016. Faster and more accurate classification of time series by exploiting a novel dynamic time warping averaging algorithm, Knowledge and Information Systems 47(1): 1-26. https://doi.org/10.1007/s10115-015-0878-8

Pishgahzadeh, B.; Soleymani, S; Faghihi, F. 2015. New active type of SFCL during unbalanced faults allocated in incoming and outgoing feeders of distribution networks, International Organization on "Technical and Physical Problems of Engineering” 7(2): 77-83.

Rahi, P. K.; Mehra, R. 2014. Analysis of power spectrum estimation using welch method for various window techniques, International Journal of Emerging Technologies and Engineering 2(6): 106-109.

Raja, M. A. Z.; Chaudhary, N. I. 2015. Two-stage fractional least mean square identification algorithm for parameter estimation of CARMA systems, Signal Processing 107: 327-339. https://doi.org/10.1016/j.sigpro.2014.06.015

Sasikiran, P.; Manohar, T. G.; Rao, S. K. 2014. Estimating the power spectrum of a wide sense stationary random process using parametric approaches (AR, MA), International Journal of Recent Advances in Engineering \& Technology 2(2): 48-53.

Selvaperumal, S. K.; Nataraj, C.; Thiruchelvam, V.; Hung, W. T. C. 2016. Speech to text synthesis from video automated subtitling using Levinson Durbin method of linear predictive coding, International Journal of Applied Engineering Research 11(4): 2388-2395.

Sykulski, A. M.; Olhede, S. C.; Lilly, J. M. 2016. A widely linear complex autoregressive process of order one, IEEE Transactions on Signal Processing 64(23): 6200-6210. https://doi.org/10.1109/TSP.2016.2599503

Širca, S. 2016. Maximum-likelihood method, in S. Širca (Ed.). Probability for Physicists, 203-225. https://doi.org/10.1007/978-3-319-31611-6_8

Wollstadt, P.; Martínez-Zarzuela, M.; Vicente, R.; Díaz-Pernas, F. J.; Wibral, M. 2014. Efficient transfer entropy analysis of nonstationary neural time series, PLoS ONE 9(7): e102833. https://doi.org/10.1371/journal.pone.0102833

Xiao, D.; Mo, F.; Zhang, Y.; Zhao, M.; Ma, L. 2018. An extended Levinson-Durbin algorithm and its application in mixed excitation linear prediction, Heliyon 4(11): e00948. http://doi.org/10.1016/j.heliyon.2018.e00948

Zabarankin, M.; Uryasev, S. 2014. Maximum likelihood method, Springer Optimization and Its Applications 85: 45-52. https://doi.org/10.1007/978-1-4614-8471-4_4

Zhuang, D. E. H.; Li, G. C. L.; Wong, A. K. C. 2014. Discovery of temporal associations in multivariate time series, IEEE Transactions on Knowledge and Data Engineering 26(12): 2969-2982. http://doi.org/10.1109/tkde.2014.2310219 